\title{
PREDICTING IMPROVEMENT OF POST-ORTHODONTIC WHITE SPOT LESIONS
}

\author{
SUSAN KIM \\ A THESIS \\ SUBMITTED IN PARTIAL FULFILLMENT OF THE \\ REQUIREMENTS FOR THE DEGREE OF
}

MASTER OF SCIENCE IN DENTISTRY

UNIVERSITY OF WASHINGTON

2015

COMMITTEE:

GREG HUANG

BURCU BAYIRLI

MEHMET SARIKAYA

Program Authorized TO OFFER DEGREE:

DEPARTMENT OF ORTHODONTICS 
(C) Copyright 2015

Susan Kim 


\title{
University of Washington
}

\begin{abstract}
Predicting Improvement of Post-Orthodontic White Spot Lesions
\end{abstract}

\author{
Susan Kim
}

\author{
Chair of the Supervisory Committee: \\ Greg J. Huang \\ Department of Orthodontics
}

\begin{abstract}
Introduction: It is well known that patients undergoing orthodontic treatment are at greater risk for developing white spot lesions (WSL). Although prevention of WSLs is always the goal, they continue to be a common sequela in patients undergoing orthodontics. For this reason, understanding the patterns of WSL improvement is of great importance to both the patient and the provider. Previous studies have shown some lesions exhibiting significant improvement, while other lesions show limited to no improvement. Our study sought to identify specific patient and tooth-related factors that are most predictive for improvement.
\end{abstract}

Methods: Patients aged 12-20 years, who had at least 1 WSL that developed during orthodontic treatment, were recruited from private dental and orthodontic offices. They all had their braces removed less than 2 months prior to enrollment into the study. Photos were taken at enrollment and 8 weeks later. Paired photographs from the 2 time points were blindly assessed for improvement in appearance by a panel of 5 dental professionals, and by 2 sets of evaluators for changes in surface area and appearance at the individual tooth level.

Results: One hundred and one subjects were included in this study. Patient age, brushing frequency, and larger percent surface area affected were associated with increased improvement of lesions. Central incisors showed greater improvement when compared to lateral incisors. Increased time since appliance removal and increased length of orthodontic treatment were associated with decreased improvement. Gender, oral hygiene status, retainer type, location of the lesion (gingival, middle, incisal), presence of staining, and lesion diffuseness were not found to be predictive for improvement.

Conclusions: Of the various patient and tooth-related factors examined; age, time since appliance removal, length of orthodontic treatment, tooth type (central vs. lateral incisor), WSL surface area, and brushing frequency had significant associations with WSL improvement. 


\section{ACKNOWLEDGEMENTS}

The author wishes to thank her research committee for their guidance in conducting this project. She is especially grateful to her committee chair, Greg Huang, who was always been so helpful in providing the necessary mentorship and feedback in order to successfully complete this project. 


\section{TABLE OF CONTENTS}

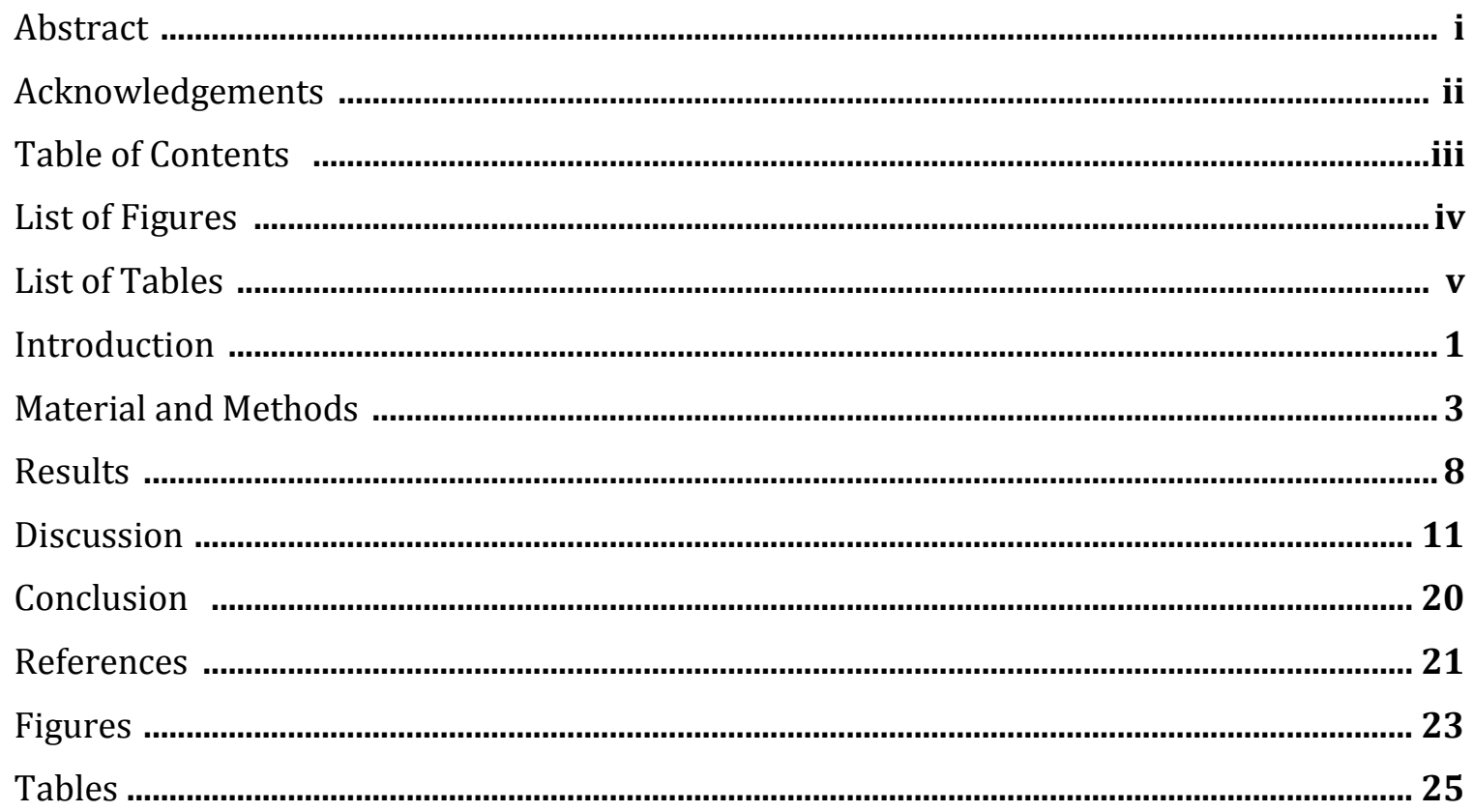




\section{LIST OF FIGURES}

\section{Figure Number}

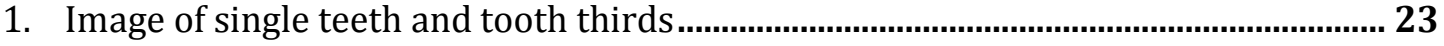

2. Appearance of a stained lesion ................................................................................... 24

3. Appearance of a diffuse, discrete, mixed lesion …..................................................... 24 


\section{LIST OF TABLES}

\section{Table Number}

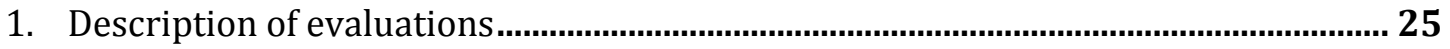

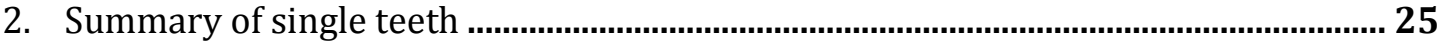

3. Summary of tooth thirds ….......................................................................................... 26

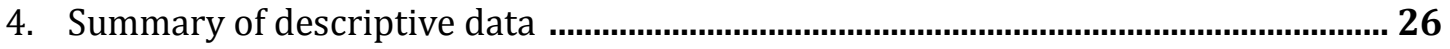

5. Subjective improvement of incisors (univariate analysis) ............................................... 27

6. Objective improvement of incisors (univariate analysis) ........................................... 27

7. Subjective improvement of incisors (multivariate analysis) …........................................ 28

8. Objective improvement of incisors (multivariate analysis) ...................................... 28

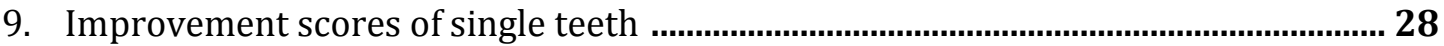

10. Improvement scores of tooth thirds …............................................................................ 29 


\section{INTRODUCTION}

Orthodontic treatment has long served as a means for providing patients with improved esthetic, functional, and psychological benefits. Unfortunately, white spot lesions (WSLs) are one of the most common and undesirable side effects that can diminish the satisfaction and joy that a patient experiences following orthodontic treatment. Some studies have shown the prevalence of WSLs to be as high as $97 \%$ among orthodontic populations ${ }^{1}$.

WSLs are characterized by their increased opacity compared to healthy enamel. They possess a whiter appearance as a result of mineral loss in the surface layers, which alters the refractory index ${ }^{2}$ and increases the scattering of light in the affected area. The appearance of the lesion can vary from an initial loss of mineral to total tooth destruction ${ }^{3}$. In some instances, stains can be incorporated into a lesion and lead to the formation of brown spots during the remineralization process, worsening the esthetic problem ${ }^{4}$.

Prevention, diagnosis, and treatment of WSLs are crucial to minimizing tooth decay and discoloration that can compromise the esthetics of a smile. It is a significant concern for both orthodontists and their patients because in addition to the risk for progressing to active disease, WSLs often affect maxillary incisors, which are generally the most visible teeth.

Several options have been proposed to address these lesions, depending upon their nature and severity. The recommended treatment can be as simple as improved home care with fluoride toothpaste for minor lesions, to more invasive options involving composite restorations. However, there is still a lack of strong evidence in the literature regarding the most effective treatment protocol ${ }^{5}$ and the ideal timing for maximizing improvement.

In addition to the abundance of available treatment options, the unpredictable patterns and degree of improvement adds to the complexity of WSL management. Often times, we see a wide range of improvement in lesions from one patient to the next. Lesions can vary in size, 
shape, and location, and are as unique as the oral environment of the patients in which they are found.

The objective of our study was to attempt to identify patient and tooth-related factors that might be predictive for WSL improvement. Knowledge of these factors might help orthodontists develop management strategies at the time braces are removed. 


\section{MATERIAL AND METHODS}

This study is a further investigation of data from a prior project that investigated WSLs. The photographs that formed the sample were originally collected from a randomized (1:1:1), single-blind, 3-arm, active controlled, parallel-group trial evaluating the improvement of WSLs for 3 different treatment arms 6 . The treatment arms compared effectiveness of MI Paste Plus (900 ppm fluoride; GC America), PreviDent fluoride varnish (22,600 ppm fluoride; Colgate Oral Pharmaceuticals, New York, NY), and usual home-care oral hygiene instruction using over-the-counter toothpaste (1100 ppm fluoride; Colgate Oral Pharmaceuticals) (control group). In the original study, photographs of WSLs were taken at two different time points: at the start of the study $\left(T_{1}\right)$ and 8 weeks later $\left(T_{2}\right)$. Data were collected from private orthodontic and general dentistry offices belonging to the Practicebased Research Collaborative in Evidence-based Dentistry network in the Northwestern United States (NW PRECEDENT). The network was co-operated by the University of Washington and the Oregon Health and Science University, and was comprised of the following states-Washington, Oregon, Montana, Idaho, and Utah.

Eligibility criteria for this study included the fulfillment of the following conditions: completion of fixed appliance orthodontic therapy within the past 2 months, the presence of at least 1 WSL on the facial surface of a maxillary incisor that was not present prior to starting orthodontic treatment, and being between the ages of 12 and 20 years. Subjects excluded from this study were those who were unwilling to be randomly assigned to 1 of the 3 treatment groups; had any abnormal oral, medical, or mental conditions; received therapy for WSLs after orthodontic treatment; displayed frank cavitations associated with the maxillary incisors; or were unable to speak or read the English language. Patients (and parents, for those under 18 years of age) consented to participate prior to the start of the study. 
All subjects completed a questionnaire, which provided us with information regarding the patient's average daily brushing frequency. Patient information such as age, gender, length of orthodontic treatment, retainer type, and oral hygiene throughout treatment was provided by their clinicians.

Two different types of evaluations (subjective and objective improvement) were performed for the 4 maxillary incisors, for each pair of photographs (initial and 8-weeks). For subjective improvement, a blinded panel of 5 dental professionals performed their assessment of improvement using a visual analog scale from 0 to $100 \mathrm{~mm}(0 \mathrm{~mm}=$ no improvement or worsened, to $100 \mathrm{~mm}=$ complete resolution of WSL). These evaluations were performed as part of the original study, and the ratings of the panel were used for overall improvement of the 4 maxillary incisors.

For objective improvement, 2 examiners (a dental student and a general dentist) performed the assessment for improvement by measuring changes in WSL surface area from each time point. WSL surface area was divided by total tooth surface area to calculate the pretreatment and posttreatment percentages of affected surface area. The change in percentage of affected surface area was obtained by subtracting posttreatment surface area from pretreatment surface area. These assessments were also performed as part of the original study for all 4 incisors.

Results from the randomized control trial found no significant difference in subjective or objective improvement in the appearance of the WSL between those who received MI Paste Plus, PreviDent fluoride varnish, and normal home care, during the 8-week period. Although some WSLs exhibited little to no improvement, many lesions did in fact show a large degree of improvement. Since the treatment arm did not appear to have a large role in the improvement of the WSL, we decided to investigate other possible factors that might be associated with the degree of WSL improvement that occurred. 
For this current study, we considered improvement of a lesion to be a visible decrease in affected surface area, minimized contrast between the WSL and surrounding healthy tooth structure, or any combination of changes resulting in an overall improved esthetic appearance. The previous study evaluated all 4 incisors as a unit, but did not evaluate each tooth individually (Table 1). In order to perform evaluations at the single tooth level, we cropped the images of the 4 maxillary incisors into individual teeth $(n=404)$ and then further cropped them into horizontal thirds, but only evaluating those portions affected $(n=728)$ (figure1). In order to maintain a uniform size and a similar level of magnification, a grid of a fixed dimension was used to reveal only the tooth or portion of the tooth being evaluated. This method allowed us to mask all other teeth and parts of the teeth, minimizing any undesired influence from the surrounding teeth on evaluation scores. Once all images were cropped to proper dimensions, lesions were then categorized according to the different characteristics we were interested in studying.

At the single tooth level, each lesion was categorized by the presence or absence of staining (Table 2). Most WSLs are uniformly white throughout (unstained), but occasionally, some WSLs display a yellowish or brownish area of discoloration (figure 2). A primary and secondary evaluator (an orthodontic resident and a general dentist) categorized staining prior to blinding for time point in order to ensure that the staining was present at the start of the study. Following categorization, time points were obscured for all images in order to reduce any expectation bias for improvement, as one may naturally expect improvement to occur over time even when no such improvement has occurred.

The images of the horizontal thirds were labeled as gingival (G), middle (M), and incisal (I) thirds (Table 3). Only portions of the tooth affected by a WSL were evaluated. Portions of the tooth not containing a lesion were not included. The same 2 evaluators independently examined each third and categorized each lesion by their diffuseness (figure 3). Any lesion 
with a discrete linear shape with areas of healthy, unaffected tooth structure adjacent to both sides of the lesion was considered to be a discrete lesion. Any lesion with a non-linear, amorphous, or ill-defined appearance was categorized as being diffuse. Any lesion containing both types of these lesions on the same tooth third was classified as being a mixed lesion. Time points were also obscured for each third, prior to evaluation.

For the evaluation of single teeth and tooth thirds, the evaluators rated each image on a WSL improvement scale of 1 to 5 as being: (1) significantly worse, (2) slightly worse, (3) the same, (4) slightly better, or (5) significantly better than its corresponding image taken at the alternative time point (Table 1). Twenty images were evaluated a second time, at least 1 month apart, in order to calculate intra-rater and inter-rater reliability.

In addition to our evaluations of images taken during the 8-week period between $\mathrm{T}_{1}-\mathrm{T}_{2}$, we also had images for 31 subjects taken on the day of appliance removal $\left(\mathrm{T}_{0}\right)$, prior to starting the study. Following appliance removal, most subjects began the study within 1 week's time $(n=73)$, but no subjects were started on the same day of appliance removal. This was to allow for natural rehydration of the enamel and to prevent excessive gingival swelling from obscuring any portion of the lesion. Of the 31 subjects who had $\mathrm{T}_{0}$ data, 4 subjects dropped out prior to completing the study. Analyzing the 27 subjects with both $\mathrm{T}_{0}$ and $\mathrm{T}_{1}$ data allowed us to evaluate the WSL improvement that occurred naturally in the first days following appliance removal, before any intervention was initiated.

\section{Statistical analysis}

Analyses were conducted using SAS software (version 9.2; SAS Institute, Cary, NC) on a Windows operating system (Microsoft).

Descriptive data were summarized with frequency tables (Table 4). Regression models were run using Generalized Estimating Equations (GEE), which allows us to account for 
clustering by site and for tooth-within-subject. ICCs for both factors were found to be negligible.

We performed univariate analyses to identify potential factors of interest (Table 5, 6), and then multivariate analyses based upon our univariate results. Models were adjusted for age, gender, time since appliance removal, length of orthodontic treatment, brushing frequency, and the original treatment arm. Although the previous study found no difference in improvement between the 3 groups, we performed a sensitivity analysis for treatment arm to verify that there were no differences between groups.

The first aim of this study was to determine if the following patient factors are predictive for the overall improvement of WSLs: age, gender, time since appliance removal, length of orthodontic treatment, self-reported tooth brushing, oral hygiene, retainer type. Each patient factor examined had the null hypothesis: no difference in WSL improvement for both subjective and objective measures.

The second aim compared the following tooth-related factors to the amount of WSL improvement: proportion of tooth surface area affected, tooth type (central or lateral incisor), presence of staining, location (gingival, middle, incisal), and lesion diffuseness. The null hypothesis was the following: no difference between each tooth-related factor and WSL improvement.

The third aim compared the amount of improvement that occurs immediately following appliance removal $\left(\mathrm{T}_{0}-\mathrm{T}_{1}\right)$ with the amount of improvement that occurs thereafter $\left(\mathrm{T}_{1}-\mathrm{T}_{2}\right)$. The null hypothesis stated there was no relationship between the amounts of improvement that occurs for each set of time points. 


\section{RESULTS}

A total of 115 subjects were eligible for evaluation in our study. Subjects were removed due to poor image quality $(n=5)$ or missing lateral incisors $(n=2)$. One subject's records were not obtainable from the previous study. Six additional subjects were dropped from the study due to being prescribed multiple retainer types. The subjects dropped from our study did not vary with respect to demographic data and initial WSL severity compared to subjects included in this study.

Intracluster correlation coefficients (ICC) were obtained using 20 sets of duplicate measurements and indicated good reliability with a value of 0.92 for the two evaluators from this study and 0.72 and 0.85 for the subjective and objective evaluators from the previous study.

A total of 101 subjects ( 49 boys and 52 girls; mean age, $14.4 \pm 1.5$ years) were included in the final analyses. Although no difference in improvement was found between the 3 treatment groups in the original randomized control trial, 28 received MI Paste Plus, 35 received PreviDent, and 38 received usual home care instruction. For those in the MI Paste Plus group, patient compliance was also factored in as part of the multivariate analysis, and was found to be insignificant.

\section{$\underline{\text { Four Maxillary Incisors }}$}

The mean subjective improvement for the 4 incisors over the 8-week period $\left(\mathrm{T}_{1}-\mathrm{T}_{2}\right)$ was $26 \%$, as determined by the expert panel. Using these subjective ratings that were collected from the original study, no patient-related factors were found to be associated with improvement (Table 7). Initial percent of surface area affected was also not significant for improvement. 
The objective surface area measurements from the original study found an average improvement of 19\% for the 4 maxillary incisors. In multivariate analyses, we found greater improvement in WSL appearance with each additional year of patient age. Time since appliance removal and length of orthodontic treatment demonstrated less improvement with each additional month (Table 8).

\section{Single Teeth}

At the single tooth level, the mean WSL improvement scale rating was 3.77 , indicating most lesions stayed the same or improved slightly over the 8-week period. Assessment at the single tooth level found lateral incisors to show less improvement when compared to central incisors. Subjects with self-reported brushing frequency of $\geq 2$ times per day were 0.42 points higher on the improvement scale than those who brushed less frequently. WSLs with a larger surface showed slightly greater improvement. Increased time since appliance removal was associated with less improvement (Table 9).

Staining of WSLs showed no relationship with improvement. Categorization for the presence of staining was done at the single tooth level and had an agreement of $87 \%$ between evaluators. Discrepancies in categorization were determined by consensus. In this study, 105 of the 404 incisors exhibited staining (26\%).

\section{Tooth Thirds}

Comparison of tooth thirds showed that gingival and middle thirds were most commonly affected, but there was no significant difference in improvement between gingival, middle, and incisal thirds. The evaluators characterized lesion diffuseness for each horizontal third, and had 98\% concordance and resolved any discrepancies in categorization by consensus. Lesion diffuseness was also not found to be related to improvement (Table 10). 


\section{Improvement by Time Point}

For subjects with $\mathrm{T}_{0}-\mathrm{T}_{1}$ data, subjective improvement for all 4 incisors was $26 \%$. Objective measurements for surface area were not measured for $\mathrm{T}_{0}$ subjects. The average WSL improvement score at the single tooth level was 3.81, indicating most teeth stayed the same or improved slightly. No patient or tooth-related factors were associated with improvement either for all 4 incisors or at the single tooth level. We found that there was no correlation between the amount of improvement that occurred between $T_{0}-T_{1}$ and $T_{1}-T_{2}(r=-0.1)$ 


\section{DISCUSSION}

We identified several factors that were associated with a significant effect on improvement in the appearance of WSLs. Overall, we saw a tendency for WSLs to improve during the 8-week period, according to all 3 evaluation methods. However, different patient and tooth-related factors were significant for improvement depending on the type of assessment.

$\underline{\text { Age }}$

With each additional year of age, there was a 3.1\% greater reduction in WSL surface area over the 8-week period. Although the range in patient age was not very large for our study (12-20 years) we can expect that with greater age, there may be some improved dexterity and ability to remove plaque and food debris with brushing. Even if patients are brushing with the same frequency and duration each day, the older patients may be more effective. It is also possible that patients who are older have a greater appreciation for the esthetics of their smile, and will make a greater effort to be diligent with oral hygiene once they become aware of their WSLs and the etiology.

When considering the effect of age on the oral environment, one study comparing the salivary content of children (6-12 years) and adults (19-44 years) found that calcium concentrations were lower in children ${ }^{7}$. Concentrations of calcium and phosphate in saliva have significant influence on the protective mechanisms of dental hard tissues within the oral environment. Since a lower salivary calcium concentration creates a higher critical $\mathrm{pH}$ and a lower driving force for remineralization, those who are younger in age may also have a saliva composition less conducive to WSL improvement. 


\section{Gender}

Studies have shown conflicting findings regarding the effect of gender on the prevalence of WSL development. There is a common belief that males tend to have decreased compliance and are also less concerned with their dental appearance ${ }^{8}$. Although some may argue that this could also affect motivation for improvement, our study found no difference in improvement between males and females.

\section{Time Since Appliance Removal}

With each additional month that elapses since appliance removal, there was $14.9 \%$ less improvement in surface area. This would suggest that the majority of improvement occurs immediately following appliance removal, so that even by the second month, improvement diminishes significantly. Some studies regarding remineralization patterns of WSLs support these findings and have described an exponential pattern of remineralization, with all improvement occurring within the first month ${ }^{9}$. This pattern of improvement is believed to be more characteristic of lesions that were experimentally induced within a few short weeks and are likely to be caused by softening of the outermost enamel layer. Other studies involving WSLs that were developed over a longer period of time, involving subsurface layers of enamel, showed the greatest amount of remineralization occurring during the first few months and then continuing at a slower rate thereafter ${ }^{10}$. As the average treatment time for our subjects was 25.7 months, the lesions were likely to have formed over a period of months rather than weeks. With that in mind, we would have expected more of a gradual tapering of improvement, but instead our findings were more consistent with that of an exponential pattern.

The average amount of subjective improvement that occurred between the day of appliance removal to start of the study $\left(\mathrm{T}_{0}-\mathrm{T}_{1}\right)$ was $26 \%$. The average time elapsed between $\mathrm{T}_{0}$ and $\mathrm{T}_{1}$ was only 4 days, so within this short period of time, without any intervention whatsoever, patients showed significant improvement. This is an important issue to consider, as baseline improvement 
must be factored into the amount of improvement that occurs after a particular form of intervention is instituted. This is especially relevant if a WSL therapy calls for application at the time of appliance removal, since the spontaneous improvement that will occur naturally should be subtracted from overall improvement.

When we looked for a correlation in subjective improvement scores between $T_{0}-T_{1}$ and $T_{1}-T_{2}$, we found no relationship between the amount of improvement that occurs early on $\left(T_{0}-T_{1}\right)$ and the amount of improvement that occurs subsequently $\left(\mathrm{T}_{1}-\mathrm{T}_{2}\right)$. We were curious to determine whether lesions that initially show a greater degree of change would show less improvement in the later weeks, since the majority of improvement may have already occurred. However, there was no particular pattern of improvement since some WSLs that improved drastically in $\mathrm{T}_{0}-\mathrm{T}_{1}$ went on to show minimal change during $\mathrm{T}_{1}-\mathrm{T}_{2}$, while others improved greatly.

\section{$\underline{\text { Treatment Time }}$}

According to objective measurements, length of orthodontic treatment showed $0.29 \%$ decreased improvement of WSLs with each additional month in treatment. Although statistically significant, a difference of $0.29 \%$ per month is not clinically significant. Even with an additional year of treatment, this only amounts to a 3.5\% decrease in improvement. One study found that teeth bonded for a relatively short treatment time (12-16 months) had the same incidence of WSLs as those with longer treatment time (up to 36 months) ${ }^{11}$. A second study found that the greatest formation of WSLs occurs in the first 6 months of treatment, with a gradual slowing occurring by 12 months ${ }^{12}$. Therefore, if lesion severity and formation are relatively established by the first year of orthodontic treatment, it is not surprising to see very minimal differences in improvement between our subjects, who were in treatment for greater than 12 months. 


\section{Tooth Brushing Frequency}

Brushing frequency of $\geq 2$ times per day was associated with an increase of 0.42 on the WSL improvement scale, when compared to those who brushed less frequently. This is not surprising, as more frequent clearing of food and plaque away from the tooth surface would decrease exposure of the enamel to the acidogenic bacteria and their products. Interestingly, in reviewing the patient survey results, we found that more subjects reported brushing $\geq 2$ times per day after appliances were removed, compared to when they were undergoing orthodontic treatment. Perhaps this increase in brushing frequency can be attributed to a heightened commitment to improving oral hygiene, since the WSLs are usually more apparent after appliances are removed.

\section{Retainer Type}

There was no significant difference in improvement attributed to the type of retainer prescribed. In terms of distribution, 55\% received Hawley retainers and $45 \%$ received vacuum-formed (Essix) retainers. Since it is believed that the free flow of saliva may be a major factor in developing WSLs ${ }^{11}$, it would be reasonable to believe that the full coverage design of an Essix retainer might inhibit some of the remineralization process from occurring. Although we do not know the compliance level of retainer wear among these subjects during the 8-week period, we can assume compliance for the Essix retainer was generally as good, if not better ${ }^{13}$ than they Hawley retainer group, due to its more esthetic design. However, improvement of the WSLs was found to be comparable between groups. Therefore, when selecting a retainer type for patients, WSL improvement need not be a primary concern for our retention protocol. 


\section{Oral Hygiene}

Surprisingly, oral hygiene did not have a significant relationship with improvement. Most patients had poor $(47 \%)$ or fair $(43 \%)$ oral hygiene throughout their orthodontic treatment. Despite having good oral hygiene, the $10 \%$ of subjects in the good hygiene group were still susceptible to WSL formation during treatment and did not exhibit greater improvement in the weeks following appliance removal. This may be due to patient factors other than oral hygiene that were not accounted for such as composition of biofilm, diet, and variations in salivary flow rate, $\mathrm{pH}$ and buffer capacity ${ }^{14}$.

\section{Tooth Type}

Lateral incisors showed less improvement compared to central incisors, which was 0.18 points lower on the improvement scale. Previous studies have shown that lateral incisors are more frequently affected with WSLs compared to central incisors ${ }^{15,16}$. Lateral incisors were also found to be the most severely affected teeth ${ }^{15}$. In comparison to the central incisor, lateral incisors are smaller in size and also have a smaller distance from bracket to free gingival margin, which is more conducive to accumulating plaque and debris ${ }^{11}$. The diminished improvement of the lateral incisors may be due to a relatively larger proportion of affected surface area and lesions that are generally more severe in nature.

\section{$\underline{\text { Location }}$}

The gingival and middle thirds made up the greatest proportion of affected sites, at $44 \%$ and $45 \%$, respectively. In comparing all potential sites, the gingival and middle thirds were affected $26 \%$ and $27 \%$ of the time, respectively. This is expected, as the areas with the greatest difficulty to cleanse are directly adjacent to and gingival to the bracket. The incisal third of the tooth comprised only $11 \%$ of the affected sites, and was affected $6 \%$ of the time. 
Although there were differences in WSL formation rates for each region of the tooth, there were no differences in their improvement.

\section{$\underline{\text { Staining }}$}

It is believed that more severe WSLs can change color from their characteristic shade of white to a brown-black color ${ }^{14}$. We examined the presence of staining to determine whether these potentially more severe WSLs would exhibit a different pattern of improvement. Of the 404 single teeth examined, 105 incisors (26\%) had staining associated with their WSLs. Our study found that the presence of staining was not related to WSL improvement, either at the single tooth level or for all 4 incisors. Those subjects with a greater number of stained teeth showed no difference in improvement compared to those with lesser or no staining present.

\section{$\underline{\text { Diffuseness }}$}

Lesion diffuseness was not a predictive factor for improvement. Eighty-eight percent of lesions were categorized as diffuse, and only $7 \%$ were discrete. The remaining proportion had some combination of both a diffuse and discrete appearance. Our objective for qualifying lesions by diffuseness and discreteness was to try and identify a lesion characteristic that can simply be evaluated by visual inspection. As lesions are so unique in appearance, it was difficult to identify a meaningful and consistent characteristic for all lesions. Although our study did not find a relationship between lesion diffuseness and improvement, future studies should attempt to identify a reliable trait that can be used.

\section{WSL Surface Area}

Although we found a positive relationship between the affected surface area and the amount of improvement, it was not a very significant amount. In order to see a 0.5 difference 
on the 5 point WSL improvement scale, it would require a $34 \%$ increase in initial WSL surface area. We would have expected to find that WSLs with less affected surface area would should greater improvement, but we did not find that to be the case. This could be related to the fact that even though a large part of the tooth's surface has developed a WSL, it does not necessarily mean that the lesion has penetrated deep into the enamel. ${ }^{14}$ Therefore, a very superficial lesion with a larger surface area could be more likely to improve compared to a deeper lesion that is confined to a smaller area. Further studies relating depth and volume of a lesion related to improvement should be performed.

One of the strengths of our study is the methods of evaluation that were utilized, as we used a panel and multiple measures of improvement looking at all 4 incisors, each single tooth, as well as tooth thirds. The visual assessment of improvement, performed by an expert panel and 2 evaluators, was an important part of evaluation, since it demonstrates changes that a clinician perceives.

Our objective measure of improvement assessed the change in percent of tooth surface affected (proportional change), which has been used in many previous studies ${ }^{15,17-20}$, rather than absolute measurements of luminance or size 21,22 . The comparison of proportional change also allows for us to account for any differences in magnification and angulations of teeth, when using photograph images.

In evaluating improvement, we noted differences between the amount of subjective and objective improvement that occurred. Subjective scores were generally higher when compared to objective changes in surface area, suggesting that improvement as we perceive is tied to other factors in addition to surface area reduction. We also speculate that a lesion must exhibit reduction beyond a certain threshold to be detected as clinical improvement. This may explain why we were able to find more predictors for improvement using 
objectives measures, compared to subjective measures. However, one might argue that the subjective measures might align better with a patient's own assessment of improvement.

A limitation of our study design is that we have combined patients who underwent three different treatment regimens for WSLs. However, the patients in each of the arms showed, on average, the same amount of improvement, and thus, the treatment arm was thought not to be the primary factor related to the amount of improvement. We also did adjust for the treatment arms in our multivariate analyses. Additionally, one common limitation to this study is that improvement may be related to lesion depth, and lesion depth is difficult to measure accurately and consistently in vivo. This is also a clinically relevant challenge since the orthodontist cannot predict the volume (depth of demineralization) of the WSL using visual cues, but they can only see the surface area. With limited information, it is difficult to correctly inform a concerned patient about the future outcome of his or her WSLs ${ }^{14}$.

Methods to accurately report lesion depth and volume, in addition to photographic images, would be ideal for future studies on improvement. Some studies have used laser fluorescence, such as DIAGNOdent (KaVo, Biberach, Germany) or quantitative light-induced fluorescence (QLF) to evaluate WSLs. Although these newer technologies are able to quantitatively assess WSLs, each method has its limitations.

With laser fluorescence technology, because these readings measure the bacterial metabolites that have penetrated into the surface deposits and porosities, they do not directly detect changes within the enamel structure ${ }^{23}$ and are not necessarily a measure of lesion size or depth. QLF is known to have a close correlation with changes in enamel structure and mineral content ${ }^{24}$ and has been validated against a number of other methods for quantification of demineralization ${ }^{25}$ in in vitro studies. Although QLF has been shown to be highly sensitive, it may not be practical for diagnosis of WSL2. A recent systematic review 
on the various detection methods concluded that for both cost and practicality considerations, visual methods should still be the standard for clinical assessment 26 .

Additional considerations for future studies would involve collection of saliva samples to test for differences in oral biofilm composition and activity, stimulated and unstimulated salivary flow rate, $\mathrm{pH}$, calcium, phosphate, and bicarbonate concentration. Lastly, details of patient diet, including snacking frequency and consumption of sugary or acidic drinks could also be evaluated. 


\section{CONCLUSION}

Of the various patient and tooth-related factors examined; age, time since appliance removal, length of orthodontic treatment, tooth type (central vs. lateral incisor), WSL surface area, and brushing frequency had significant associations with WSL improvement. Gender, oral hygiene status, retainer type, location of the lesion (gingival, middle, incisal), presence of staining, and lesion diffuseness were not found to be predictive for improvement. 


\section{REFERENCES}

1. Boersma JG, van der Veen MH, Lagerweij MD, Bokhout B, Prahl-Andersen B. Caries prevalence measured with QLF after treatment with fixed orthodontic appliances: influencing factors. Caries Res 2005;39(1):41-7.

2. Heymann GC, Grauer D. A contemporary review of white spot lesions in orthodontics. J Esthet Restor Dent 2013;25(2):85-95.

3. Kidd EA, Fejerskov O. What constitutes dental caries? Histopathology of carious enamel and dentin related to the action of cariogenic biofilms. J Dent Res 2004;83 Spec No C:C35-8.

4. Gugnani N, Pandit IK, Gupta M, Josan R. Caries infiltration of noncavitated white spot lesions: A novel approach for immediate esthetic improvement. Contemp Clin Dent 2012;3(Suppl 2):S199-202.

5. Chen H, Liu X, Dai J, et al. Effect of remineralizing agents on white spot lesions after orthodontic treatment: a systematic review. Am J Orthod Dentofacial Orthop 2013;143(3):376-82 e3.

6. Huang GJ, Roloff-Chiang B, Mills BE, et al. Effectiveness of MI Paste Plus and PreviDent fluoride varnish for treatment of white spot lesions: a randomized controlled trial. Am J Orthod Dentofacial Orthop 2013;143(1):31-41.

7. Anderson P, Hector MP, Rampersad MA. Critical $\mathrm{pH}$ in resting and stimulated whole saliva in groups of children and adults. Int J Paediatr Dent 2001;11(4):266-73.

8. Vallittu PK, Vallittu AS, Lassila VP. Dental aesthetics--a survey of attitudes in different groups of patients. J Dent 1996;24(5):335-8.

9. Ogaard B, Ten Bosch JJ. Regression of white spot enamel lesions. A new optical method for quantitative longitudinal evaluation in vivo. Am J Orthod Dentofacial Orthop 1994;106(3):238-42.

10. Al-Khateeb S, Forsberg CM, de Josselin de Jong E, Angmar-Mansson B. A longitudinal laser fluorescence study of white spot lesions in orthodontic patients. Am J Orthod Dentofacial Orthop 1998;113(6):595-602.

11. Gorelick L, Geiger AM, Gwinnett AJ. Incidence of white spot formation after bonding and banding. Am J Orthod 1982;81(2):93-8.

12. Tufekci E, Dixon JS, Gunsolley JC, Lindauer SJ. Prevalence of white spot lesions during orthodontic treatment with fixed appliances. Angle Orthod 2011;81(2):20610.

13. Pratt MC, Kluemper GT, Lindstrom AF. Patient compliance with orthodontic retainers in the postretention phase. Am J Orthod Dentofacial Orthop 2011;140(2):196-201.

14. Torlakovic L, Olsen I, Petzold C, Tiainen H, Ogaard B. Clinical color intensity of white spot lesions might be a better predictor of enamel demineralization depth than traditional clinical grading. Am J Orthod Dentofacial Orthop 2012;142(2):191-8.

15. Chapman JA, Roberts WE, Eckert GJ, Kula KS, Gonzalez-Cabezas C. Risk factors for incidence and severity of white spot lesions during treatment with fixed orthodontic appliances. Am J Orthod Dentofacial Orthop 2010;138(2):188-94.

16. Sonesson M, Twetman S, Bondemark L. Effectiveness of high-fluoride toothpaste on enamel demineralization during orthodontic treatment--a multicenter randomized controlled trial. Eur J Orthod 2013.

17. Willmot DR. White lesions after orthodontic treatment: does low fluoride make a difference? J Orthod 2004;31(3):235-42; discussion 02. 
18. Wu G, Liu X, Hou Y. Analysis of the effect of CPP-ACP tooth mousse on enamel remineralization by circularly polarized images. Angle Orthod 2010;80(5):933-8.

19. Benson PE, Shah AA, Willmot DR. Measurement of white lesions surrounding orthodontic brackets: captured slides vs digital camera images. Angle Orthod 2005;75(2):226-30.

20. Kanthathas K, Willmot DR, Benson PE. Differentiation of developmental and postorthodontic white lesions using image analysis. Eur J Orthod 2005;27(2):167-72.

21. Livas C, Kuijpers-Jagtman AM, Bronkhorst E, Derks A, Katsaros C. Quantification of white spot lesions around orthodontic brackets with image analysis. Angle Orthod 2008;78(4):585-90.

22. Benson PE, Ali Shah A, Robert Willmot D. Polarized versus nonpolarized digital images for the measurement of demineralization surrounding orthodontic brackets. Angle Orthod 2008;78(2):288-93.

23. Pretty IA. Caries detection and diagnosis: novel technologies. J Dent 2006;34(10):727-39.

24. Aljehani A, Tranaeus S, Forsberg CM, Angmar-Mansson B, Shi XQ. In vitro quantification of white spot enamel lesions adjacent to fixed orthodontic appliances using quantitative light-induced fluorescence and DIAGNOdent. Acta Odontol Scand 2004;62(6):313-8.

25. Pretty IA, Pender N, Edgar WM, Higham SM. The in vitro detection of early enamel de- and re-mineralization adjacent to bonded orthodontic cleats using quantitative light-induced fluorescence. Eur J Orthod 2003;25(3):217-23.

26. Gomez J, Tellez M, Pretty IA, Ellwood RP, Ismail AI. Non-cavitated carious lesions detection methods: a systematic review. Community Dent Oral Epidemiol 2013;41(1):54-66. 
FIGURES

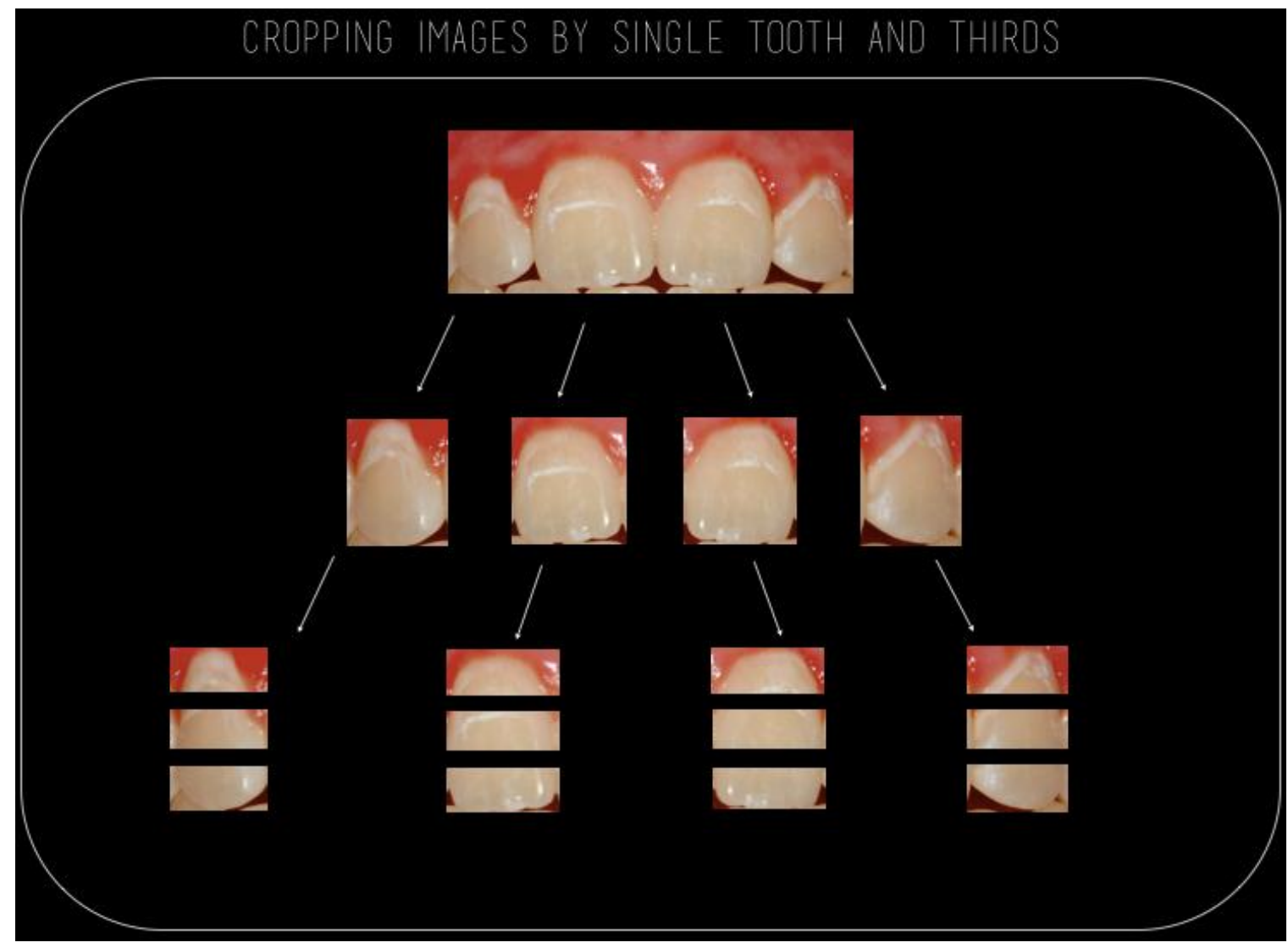

Figure 1. Images of 4 incisors, single teeth, and tooth thirds. 


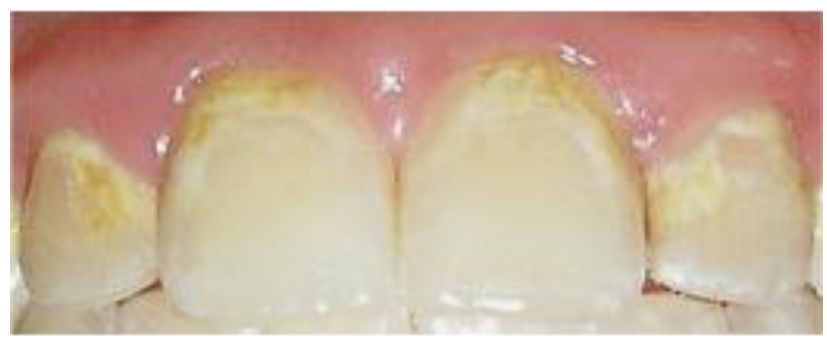

Figure 2. Example of a WSL displaying staining.
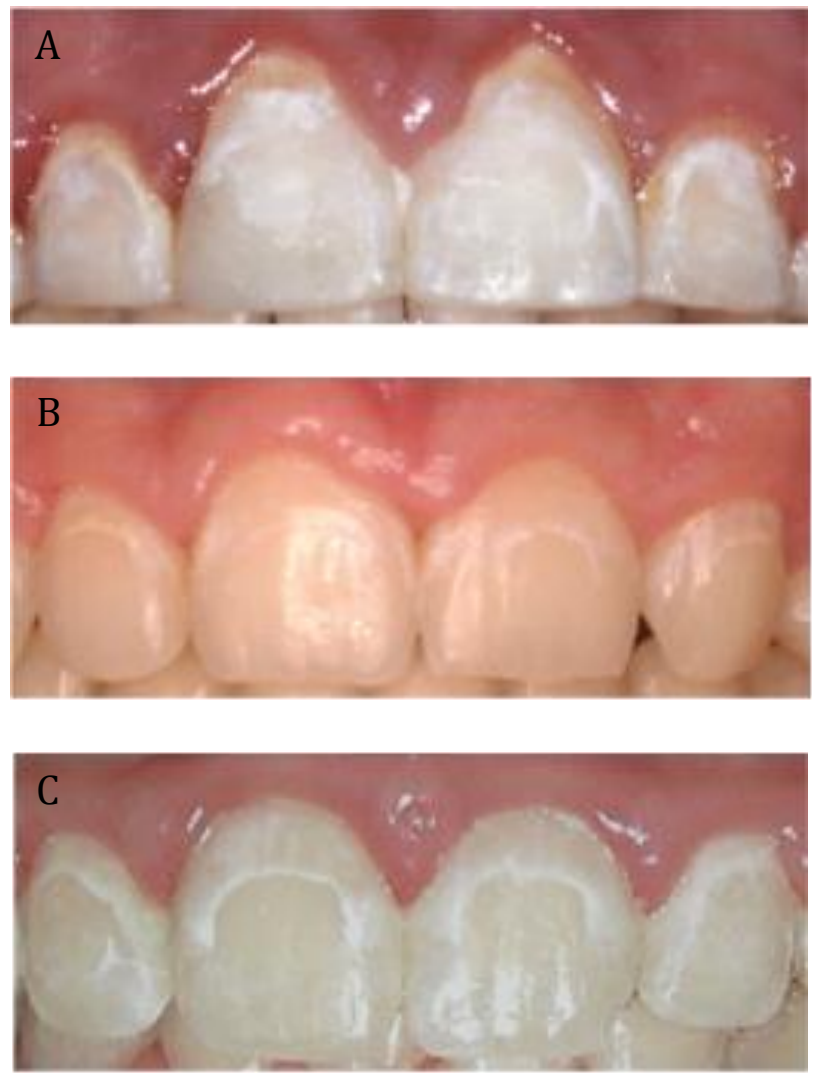

Figure 3. Examples of a A) diffuse lesion, B) discrete lesion, C) mixed lesion. 
TABLES

\begin{tabular}{|c|c|c|c|}
\hline Evaluation Level & $\begin{array}{c}\text { Evaluation } \\
\text { Type }\end{array}$ & Outcome Measure & Factors Evaluated \\
\hline \multirow[b]{2}{*}{$\begin{array}{l}4 \text { Maxillary } \\
\text { Incisors }\end{array}$} & $\begin{array}{l}\text { Subjective } \\
\text { Improvement }\end{array}$ & \% Visual improvement & \multirow{2}{*}{$\begin{array}{l}\text {-Age } \\
\text {-Gender } \\
\text {-Time since deband } \\
\text {-Treatment time } \\
\text {-Brushing frequency } \\
\text {-Oral hygiene } \\
\text {-Retainer type } \\
\text {-Initial WSL surface area }\end{array}$} \\
\hline & $\begin{array}{l}\text { Objective } \\
\text { Improvement }\end{array}$ & $\%$ Reduction of surface area & \\
\hline Single Tooth & $\begin{array}{l}\text { Improvement } \\
\text { Scale }\end{array}$ & $\begin{array}{l}\text { 1: Significantly worse } \\
\text { 2: Slightly worse } \\
\text { 3: Same } \\
\text { 4: Slightly better } \\
\text { 5: Significantly better }\end{array}$ & $\begin{array}{l}\text {-Age } \\
\text {-Gender } \\
\text {-Time since deband } \\
\text {-Treatment time } \\
\text {-Brushing frequency } \\
\text {-Oral hygiene } \\
\text {-Retainer type } \\
\text {-Initial WSL surface area } \\
\text {-Tooth type } \\
\text {-Staining }\end{array}$ \\
\hline Tooth Thirds & $\begin{array}{l}\text { Improvement } \\
\text { Scale }\end{array}$ & $\begin{array}{l}\text { 1: Significantly worse } \\
\text { 2: Slightly worse } \\
\text { 3: Same } \\
\text { 4: Slightly better } \\
\text { 5: Significantly better }\end{array}$ & $\begin{array}{l}\text {-Age } \\
\text {-Gender } \\
\text {-Time since deband } \\
\text {-Treatment time } \\
\text {-Brushing frequency } \\
\text {-Oral hygiene } \\
\text {-Retainer type } \\
\text {-Initial WSL surface area } \\
\text {-Lesion location } \\
\text {-Diffuseness }\end{array}$ \\
\hline
\end{tabular}

\begin{tabular}{|l|l|}
\hline Table 2: Summary of Single Teeth $(\mathbf{n = 4 0 4 )}$ & $202(50 \%)$ \\
\hline Central Incisors & $105(26 \%)$ \\
\hline Staining & $11.6(10.6)$ \\
\hline Percent initial surface area affected (SD) \\
\hline
\end{tabular}




\begin{tabular}{|l|c|}
\hline Table 3: Summary of Tooth Thirds $(\mathbf{n = 7 2 8 )}$ & \\
\hline Location of Lesion & $320(44.0 \%)$ \\
\hline Gingival & $331(45.5 \%)$ \\
\hline Middle & $77(10.6 \%)$ \\
\hline Incisal & $640(87.9 \%)$ \\
\hline Lesion Diffuseness & $50(6.9 \%)$ \\
\hline Diffuse & $38(5.2 \%)$ \\
\hline Discrete & \\
\hline Mixed & \\
\hline
\end{tabular}

\begin{tabular}{|l|c|}
\hline Table 4: Summary of Descriptive Data $(\mathbf{n}=\mathbf{1 0 1})$ & $14.4(1.5)$ \\
\hline Age in years (SD) & $52(51.5 \%)$ \\
\hline Female & $0.107(0.077)$ \\
\hline Percent initial surface area affected (SD) & $10(9.9 \%)$ \\
\hline Oral Hygiene & $43(42.6 \%)$ \\
\hline Good & $48(47.5 \%)$ \\
\hline Fair & $0.26(0.44)$ \\
\hline Poor & $73(72.3 \%)$ \\
\hline Months since appliance removal (SD) & $25.7(9.7)$ \\
\hline Mean (SD) & \\
\hline$\leq 1$ week & $37(36.6 \%)$ \\
\hline Months in orthodontic treatment (SD) & $64(63.4 \%)$ \\
\hline Brushing Frequency & \\
\hline$\leq 1$ time per day & $56(55.5 \%)$ \\
\hline$\geq 2$ times per day & $45(44.6 \%)$ \\
\hline Retainer style & \\
\hline Hawley & \\
\hline Essix & \\
\hline
\end{tabular}




\begin{tabular}{|c|c|c|c|c|}
\hline \multirow[b]{2}{*}{ Age } & \multirow{2}{*}{$\begin{array}{c}\text { Improvement (\%) } \\
0.3\end{array}$} & \multicolumn{2}{|c|}{$95 \% C I$} & \multirow{2}{*}{$\begin{array}{c}\text { Pvalue } \\
0.85\end{array}$} \\
\hline & & -2.9 & 3.5 & \\
\hline \multicolumn{5}{|l|}{ Gender } \\
\hline Female & 0.6 & -7.7 & 8.9 & 0.89 \\
\hline Male & - & - & - & - \\
\hline Removal time (months) & -2.9 & -9.4 & 3.5 & 0.38 \\
\hline Treatment time (months) & -0.04 & -0.4 & 0.3 & 0.80 \\
\hline \multicolumn{5}{|l|}{ Brushing Frequency } \\
\hline$\leq 1$ time per day & - & - & - & - \\
\hline$\geq 2$ times per day & 6.2 & -4.7 & 17.0 & 0.27 \\
\hline WSL surface area & -16.0 & -86.6 & 54.6 & 0.66 \\
\hline \multicolumn{5}{|l|}{ Retainer Type } \\
\hline Hawley & - & - & - & - \\
\hline Essix & 0.8 & -6.0 & 7.6 & 0.81 \\
\hline \multicolumn{5}{|l|}{ Oral Hygiene } \\
\hline Good & 2.6 & -3.1 & 8.3 & 0.38 \\
\hline Fair & 2.2 & -5.8 & 10.2 & 0.59 \\
\hline Poor & - & - & - & - \\
\hline
\end{tabular}

\begin{tabular}{|l|c|c|c|c|}
\hline \multicolumn{1}{|l|}{ Table 6: Objective Improvement of 4 Maxillary Incisors (univariate analysis) } \\
\hline Age & Improvement (\%) & \multicolumn{2}{|c|}{$95 \%$ CI } & P value \\
\hline Gender & 1.3 & -1.4 & 4.0 & 0.34 \\
\hline Female & & & & 0.73 \\
\hline Male & 1.5 & -7.1 & 10.2 & - \\
\hline Removal time (months) & - & - & - & $<.0001$ \\
\hline Treatment time (months) & -11.5 & -16.6 & -6.4 & 0.09 \\
\hline Brushing Frequency & -0.3 & -0.6 & 0.04 & - \\
\hline$\leq 1$ time per day & & & & 0.14 \\
\hline$\geq 2$ times per day & - & - & - & 0.93 \\
\hline WSL surface area & 7.4 & -2.4 & 17.2 & \\
\hline Retainer Type & 2.6 & -59.6 & 64.8 & - \\
\hline Hawley & - & & & 0.34 \\
\hline Essix & -3.4 & -10.6 & 3.7 & \\
\hline Oral Hygiene & -0.1 & -7.3 & 7.2 & \\
\hline Good & 5.3 & -1.2 & 11.7 & 0.99 \\
\hline Fair & - & - & - & 0.11 \\
\hline Poor & & & & - \\
\hline
\end{tabular}




\begin{tabular}{|l|c|c|c|c|}
\hline Table 7: Subjective Improvement of 4 Maxillary Incisors (multivariate analysis) \\
\hline & Improvement (\%) & \multicolumn{2}{|c|}{$95 \%$ CI } & P value \\
\hline Age & 0.5 & -2.5 & 3.6 & 0.72 \\
\hline Gender & & & & \\
\hline Female & -0.6 & -9.3 & 8.1 & - \\
\hline Male & - & - & - & 0.90 \\
\hline Removal time (months) & -4.3 & -10.9 & 2.3 & 0.97 \\
\hline Treatment time (months) & -0.01 & -0.3 & 0.3 & - \\
\hline Brushing Frequency & & & & - \\
\hline Brushing $\leq 1$ time per day & - & - & - & 0.29 \\
\hline Brushing $\geq 2$ times per day & 6.6 & -5.6 & 18.8 & \\
\hline
\end{tabular}

\begin{tabular}{|l|c|c|c|c|}
\hline \multicolumn{2}{|l|}{ Table 8: Objective Improvement of 4 Maxillary Incisors (multivariate analysis) } \\
\hline & Improvement (\%) & \multicolumn{2}{|c|}{$95 \%$ CI } & P value \\
\hline Age & 3.1 & 0.5 & 5.8 & 0.02 \\
\hline Gender & & & & 0.83 \\
\hline Female & 0.8 & -6.6 & 8.3 & - \\
\hline Male & - & - & - & $<0.0001$ \\
\hline Removal time (months) & -14.9 & -20.6 & -9.2 & 0.04 \\
\hline Treatment time (months) & -0.3 & -0.6 & -0.02 & \\
\hline Brushing Frequency & & & & - \\
\hline Brushing $\leq 1$ time per day & - & - & - & 0.15 \\
\hline Brushing $\geq 2$ times per day & 7.5 & -2.8 & 17.8 & \\
\hline
\end{tabular}

\begin{tabular}{|l|c|c|c|c|}
\hline \multicolumn{1}{|l|}{ Table 9: Improvement Scores of Single Teeth (multivariate analysis) } \\
\hline Age & Improvement & \multicolumn{2}{|c|}{$95 \%$ CI } & P value \\
\hline Gender & 0.01 & -0.08 & 0.1 & 0.84 \\
\hline Female & & & & 0.44 \\
\hline Male & -0.09 & -0.3 & 0.1 & - \\
\hline Removal time (months) & - & - & - & $<0.0001$ \\
\hline Treatment time (months) & -0.64 & -0.9 & -0.4 & 0.81 \\
\hline Brushing Frequency & -0.002 & -0.02 & 0.01 & - \\
\hline Brushing $\leq 1$ time per day & & & & 0.03 \\
\hline Brushing $\geq 2$ times per day & - & - & - & \\
\hline Tooth Type & 0.42 & 0.03 & 0.81 & - \\
\hline Central Incisor & & & & 0.03 \\
\hline Lateral Incisors & - & - & - & 0.02 \\
\hline WSL surface area & -0.18 & -0.3 & -0.02 & \\
\hline Staining & 0.01 & 0.003 & 0.03 & \\
\hline
\end{tabular}




\begin{tabular}{|l|c|c|c|c|}
\hline \multicolumn{1}{|l|}{ Table 10: Improvement Scores of Tooth Thirds (multivariate analysis) } \\
\hline Age & Improvement & \multicolumn{2}{|c|}{$95 \%$ CI } & P value \\
\hline Gender & -0.01 & -0.1 & 0.1 & 0.72 \\
\hline Female & & & & 0.62 \\
\hline Male & -0.05 & -0.2 & 0.1 & - \\
\hline Removal time (months) & - & - & - & 0.08 \\
\hline Treatment time (months) & -0.2 & -0.5 & 0.03 & 0.39 \\
\hline Brushing Frequency & -0.004 & -0.01 & 0.005 & - \\
\hline Brushing $\leq 1$ time per day & & & & 0.02 \\
\hline Brushing $\geq 2$ times per day & - & - & - & \\
\hline Location & 0.3 & 0.1 & 0.5 & 0.69 \\
\hline Gingival & & & & - \\
\hline Middle & -0.03 & -0.2 & 0.1 & 0.10 \\
\hline Incisal & - & - & - & \\
\hline Diffuseness & -0.2 & -0.5 & 0.05 & \\
\hline Diffuse & & & & 0.97 \\
\hline Discrete & -0.01 & -0.3 & 0.3 & - \\
\hline Mixed & - & - & - & \\
\hline
\end{tabular}

